\section{NOVA TELLVS}

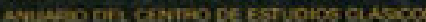

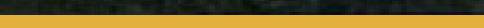

.
Nova Tellus

ISSN: 0185-3058

novatelu@servidor.unam.mx

Centro de Estudios Clásicos

México

SALLES, Ricardo

Sobre la atribución a Crisipo de una teoría estoica de la responsabilidad moral

Nova Tellus, vol. 23, núm. 2, 2005, pp. 101-124

Centro de Estudios Clásicos

Distrito Federal, México

Disponible en: http://www.redalyc.org/articulo.oa?id=59120933004

- Cómo citar el artículo

- Número completo

- Más información del artículo

Página de la revista en redalyc.org

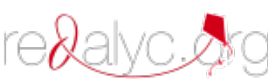

Sistema de Información Científica

Red de Revistas Científicas de América Latina, el Caribe, España y Portugal

Proyecto académico sin fines de lucro, desarrollado bajo la iniciativa de acceso abierto 


\title{
Sobre la atribución a Crisipo de una teoría estoica de la responsabilidad moral
}

\author{
Ricardo SALLES \\ Universidad Nacional Autónoma de México \\ rsalles@servidor.unam.mx
}

RESUMEN: Este artículo pone en duda la tesis de que la teoría de la responsabilidad moral descrita en Alejandro de Afrodisia, De Fato, 13, y en Nemesio de Emesa, De Natura Hominis, 35, tiene como autor a un estoico tardío, probablemente Filópator ( $f l$. primera mitad del siglo II d. C.). Esta tesis ha sido defendida recientemente por algunos estudiosos prominentes de la historia del estoicismo, entre ellos, por Susanne Bobzien en su reciente libro Determinism and Freedom in Stoic Philosophy. Por el contrario, se intenta establecer que los argumentos que ella ofrece a favor de esta atribución tardía son inadecuados, y se sostiene que el autor de la teoría pudo haber sido Crisipo (c. 280-c. 206 a. C.), tercer escolarca de la Stoa.

$$
* * *
$$

ABSTRACT: This article casts doubt on the thesis that the Stoic author of the theory of moral responsibility reported in Alexander of Aphrodisias, De Fato, 13, and Nemesius of Emesa, De Natura Hominis, 35, is late. In particular, it argues -against an interpretation recently revived by Susanne Bobzien in Determinism and Freedom in Stoic Philosophy - that this author cannot be Philopator (fl. first half of second century A.D.). Bobzien's arguments in favour of this late attribution are questionable: there is good reason for thinking that the real author of this doctrine is Chrysippus (c. 280-c. 206 B.C), the third head of the Stoic school.

Palabras Clave: alejandro-de-afrodisia, crisipo, estoicismo, filópator, moral, nemesio-de-emesa, responsabilidad, teoría.

RECEPCIÓN: 19 de octubre de 2004.

ACEPTACIÓN: 11 de mayo de 2005. 



\title{
Sobre la atribución a Crisipo de una teoría estoica de la responsabilidad moral
}

\author{
Ricardo SALLES
}

El objetivo de este artículo es defender la atribución a Crisipo de la teoría estoica de la responsabilidad moral descrita por Alejandro de Afrodisia en el capítulo 13 de De Fato $(F)$, y por Nemesio de Emesa en el capítulo 35 de De Natura Hominis $(N)$. Aunque Alejandro no se refiere por su nombre al autor de esta teoría, a la que llamaré ' $C$ ', Nemesio la atribuye a Crisipo y a otros estoicos: 'quienes afirman esto (son Crisipo, Filópator y otros muchos y famosos estoicos)...' ( $N, 105$, 12-13). ${ }^{1}$ Esto representa una prueba de que Crisipo es efectivamente el autor de la teoría, a la vez que constituye una buena razón para pensar que los otros estoicos a los que se refiere el texto aceptaron sus ideas y se limitaron a seguirlo. Sin embargo, a pesar de esto, algunos estudiosos recientes han puesto esta atribución en duda. Pienso que son cuestionables las razones que ellos aducen para hacerlo, y pretendo ofrecer una serie de argumentos que permitan ver claramente por qué son cuestionables.

\section{La controversia en torno a la autoría de $\mathrm{C}$}

En tiempos recientes, la atribución de $C$ ha generado una amplia controversia erudita. No se ha puesto en duda que la

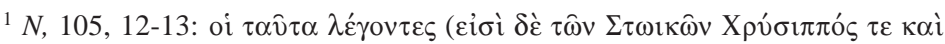

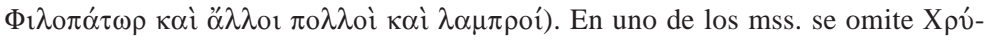

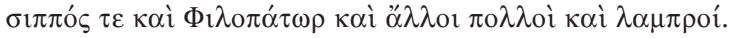


teoría sea estoica. La disputa ha versado, más bien, sobre si se trata de una teoría temprana y, muy particularmente, crisipia, o se trata, más bien, de una doctrina tardía dentro del estoicismo. Cabe distinguir tres líneas interpretativas generales:

(A) $C$ es una teoría que el propio Crisipo diseñó. Si se hace referencia a Filópator et al. en $N$, es porque las ideas crisipias que figuran en $C$ perduraron sin mayores alteraciones al menos hasta la época de Filópator (primera mitad del siglo segundo d. C.). Debe reconocerse que $C$ difiere en varios puntos importantes de otras teorías compatibilistas que sabemos que pertenecen a Crisipo. Sin embargo, estas diferencias se deben, no al hecho de que sean teorías rivales, sino a que se complementan la una con la otra.

(B) Es probable que $C$, tal como se formula en $F, 13$ y $N, 35$, no sea una teoría que haya diseñado Crisipo. No obstante, $C$ no contiene ninguna idea que no esté ya en potencia en el estoicismo temprano de Crisipo. Por consiguiente, el verdadero autor de $C$ (que pudo haber sido Filópator) no hizo ninguna innovación sustantiva dentro del pensamiento estoico sobre la responsabilidad moral.

De estas líneas interpretativas, la primera es la más común. La lista de quienes la han adoptado incluye a von Arnim, ${ }^{2}$ Long, ${ }^{3}$ Amand, ${ }^{4}$ Reesor, ${ }^{5}$ y Botros. ${ }^{6}$ La segunda ha sido defendida por Sharples, ${ }^{7}$ Frede, ${ }^{8}$ e Inwood. ${ }^{9}$

Pero, en marcado contraste con (A) y (B), existe una tercera línea interpretativa que ha sido defendida por $\operatorname{Theiler}^{10} \mathrm{y}$, muy

\footnotetext{
${ }^{2}$ Esto lo sugiere su decisión de incluir los pasajes relevantes de $F, 13$ y $N, 35$ entre los 'Chrysippi Fragmenta' de SVF (II, 979 y II, 991, respectivamente).

${ }^{3}$ Cf. Long (1970), 268, n. 4 y (1971), 180, n. 27.

${ }^{4}$ Cf. Amand (1973), 560-564.

${ }^{5}$ Cf. Reesor (1978), 187.

${ }^{6}$ Cf. Botros (1995), esp. 283.

${ }^{7}$ Cf. Sharples (1978), 253-256, (1991), 191 y (2001), 552.

${ }^{8}$ Cf. Frede (1982), 276-277.

${ }^{9}$ Cf. Inwood (1985), 88-91.

${ }^{10} \mathrm{Cf}$. Theiler (1946).
} 
recientemente, por Bobzien, ${ }^{11}$ quien le ha dado un nuevo ímpetu:

(C) $C$ no puede atribuirse de ninguna manera a Crisipo. Se trata de una teoría que refleja ideas sobre la responsabilidad moral, que, si bien son estoicas, difieren sustancialmente de las de Crisipo. $C$ fue diseñada por estoicos tardíos, y en especial por Filópator, con el objetivo de responder a objeciones que formularon filósofos anti-deterministas del platonismo medio hacia fines del siglo primero o principios del segundo de nuestra era, quienes encauzaron la discusión en torno al determinismo causal y la responsabilidad moral hacia una nueva dirección, ajena a las disputas entre los estoicos tempranos y sus rivales.

En lo que sigue, ofreceré razones para dudar de esta interpretación extrema. Si bien es imposible establecer con certeza que Crisipo fue efectivamente el autor de $C$, existen motivos dialécticos y filosóficos para creer que (A) es la interpretación correcta, o, al menos, para rechazar (C).

Antes de comenzar, deseo examinar dos razones preliminares que se han usado para cuestionar el origen crisipio de la teoría: la presencia del nombre de Filópator en $N, 35$ y la ausencia del nombre de Crisipo en $F, 13$.

Se ha considerado que la referencia en $N, 35$ a Filópator y su tratado Peri heimarmenês constituye una razón (entre muchas otras, de las cuales me ocupo más adelante) para negar la autoría de Crisipo. ${ }^{12}$ El argumento es el siguiente: (i) Filópator es un estoico oscuro de inicios del siglo segundo d. C. (su floruit se sitúa entre el año 80 y el 140), a diferencia de Crisipo, quien era mucho más famoso que él, incluso en el siglo segundo. Por lo tanto, (ii) Nemesio no habría mencionado el nombre de Filópator junto con el de Crisipo, si la fuente que usó Nemesio

\footnotetext{
${ }^{11}$ Cf. Bobzien (1998.1) y (1998.2), capítulo 8.
}

${ }^{12}$ Cf. Bobzien (1998.2), 368-369. 
hubiese sido una obra de Crisipo. Si esto es así, (iii) la fuente que usó Nemesio fue el tratado de Filópator, a cuyo título se

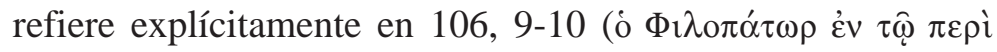

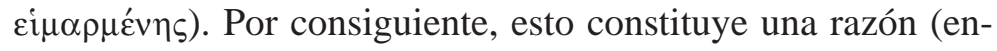
tre otras) para pensar que (iv) es Filópator, y no Crisipo, el autor de $C$. Dado este argumento, se necesita explicar por qué Nemesio menciona a Crisipo. La explicación que se ofrece es que (v) en su Peri heimarmenês, Filópator se presenta a sí mismo como un seguidor de Crisipo, aunque lo hace, no porque realmente siga sus ideas de cerca, sino porque desea realzar el estatus de su propias ideas, asociándolas con un nombre famoso, aun cuando las ideas que él presenta de este modo difieran sustancialmente de las de Crisipo.

El argumento me parece persuasivo hasta su etapa (iii). Pero la inferencia de (iii) a (iv) carece de fundamento porque (v) es cuestionable. Suponiendo que el Peri heimarmenês de Filópator es efectivamente la fuente que usó Nemesio, el hecho de que este último incluya a Crisipo en la lista de quienes han defendido $C$, pudo deberse a la presencia, en ese tratado, de citas a la obra de Crisipo e, incluso, de una referencia explícita a su autoría. En tal caso, es muy posible que el tratado de Filópator haya contenido ideas que él mismo, junto con otros estoicos importantes, aceptara sin ningún tipo de reserva. Esta opción puede explicar de modo satisfactorio por qué Nemesio se refiere a $C$ como una teoría que defienden 'Crisipo y Filópator y otros muchos y famosos <estoicos>'. Por consiguiente, la presencia del nombre de Filópator en esta enumeración no constituye un buen motivo para pensar que $C$ no es crisipia.

La ausencia de toda referencia explícita a Crisipo en el texto de $F, 13$ también se ha considerado como una razón contra su autoría. ${ }^{13}$ Se ha considerado a esto como una razón por dos motivos. En primer lugar, existen obras de Alejandro

\footnotetext{
${ }^{13}$ Cf. Bobzien (1998.2), 369.
} 
en las cuales se menciona a Crisipo por su nombre cuando Alejandro discute expresamente sus ideas. Un ejemplo de esto es el tratado De Mixtione. ${ }^{14}$ Por lo tanto, si las ideas que Alejandro discute en $F, 13$, fuesen crisipias, Alejandro habría mencionado su nombre. En segundo lugar, en la antigüedad no se solía citar el nombre de una persona cuando se le criticaba en vida, lo cual es una convención que Alejandro frecuentemente siguió. ${ }^{15}$ Por lo tanto, las ideas que Alejandro describe en el capítulo 13 de $F$, y que critica en el capítulo 14 , deben pertenecer a una escuela estoica contemporánea de Alejandro y no al propio Crisipo.

En contra de estos argumentos, es preciso advertir que en $F$ Alejandro no se refiere abiertamente ni siquiera a los estoicos. Por lo tanto, el hecho de que no se mencione a Crisipo por su nombre no prueba que él no sea su autor. Y en realidad hay una buena razón por la cual Alejandro no se refiere abiertamente a los estoicos, que tiene que ver con el contexto político en el cual el tratado fue escrito. Como lo señala Thillet en la introducción a su edición del texto, ${ }^{16}$ el tratado está dedicado a los emperadores romanos Séptimo Severo y Carácala (164, 3), quienes eran admiradores de Marco Aurelio y simpatizaban con la filosofía estoica en general. La dedicatoria es probablemente una señal de que la intención de Alejandro era agradarlos a cambio de habérsele otorgado la cátedra de filosofía aristotélica en la escuela de Atenas. Atacar abiertamente a los estoicos sería ir en contra de sus propias intenciones e intereses en esas circunstancias particulares. Esto puede explicar por qué Alejandro no menciona por su nombre ni a los estoicos en general ni a ningún estoico en particular. Pero esto no impide que, como quiere la interpretación (C), Crisipo haya sido el autor de $C$.

\footnotetext{
${ }^{14}$ Cf., por ejemplo, mixt, 213, 7; 216, 8 y 14. Para otros ejemplos, cf. Sharples (1983), 19, n. 120

${ }^{15}$ Para este asunto, cf. también D. Frede (1982), 277.

16 Thillet (1984), lxxviii y lxxxii-xc.
} 
En resumidas cuentas, la presencia del nombre de Filópator en $N$, 35, y la ausencia del de Crisipo en $F, 13$, no prueban que $C$ sea tardía.

\section{Las diferencias entre C y otra teoría crisipia de la responsabilidad}

Existen importantes diferencias entre $C$ y lo que sabemos con cierta certeza de otras fuentes acerca del pensamiento de Crisipo sobre la responsabilidad moral. Entre dichas fuentes se encuentran Cicerón, De Fato (DF), 41-43, y Aulo Gelio, Noctes Atticae (NA), VII, ii, 11, las cuales describen con cierto detalle una teoría de Crisipo a la que llamaré ' $C$ '. Ahora bien, podría verse en estas diferencias entre $C$ y $C^{*}$ una razón para negar que Crisipo sea el autor de $C$, pues ¿cómo podría ser Crisipo el autor de dos teorías tan distintas? En contra de este argumento, mostraré que, si examinamos con cuidado las diferencias, podemos ver que no revelan ningún desacuerdo sustantivo, que impidiera que las dos teorías tuvieran un mismo autor. Por lo tanto, no representan razón alguna para descartar que Crisipo sea el autor de ambas.

(1) En primer lugar, hallamos en $C$ una formulación clara de un principio que podríamos llamar de 'regularidad de la experiencia', el cual atribuye universalmente las mismas causas a los mismos efectos. Tal principio está ausente de $C^{*}$. Pero si bien esto es así, es claro que en el estoicismo temprano ya está el concepto mismo de un determinismo basado en regularidades. En efecto, sabemos que la doctrina temprana del eterno retorno implica un tipo de determinismo que descansa sobre regularidades. ${ }^{17}$ Realicemos una comparación detallada entre $C$ y esa doctrina con el propósito de ver cómo ambas se basan en el principio.

${ }^{17}$ Cf. Salles (2003). Véase también Gould (1974), 18-9 y Sharples (1978), 256. 
Una tesis clave de $C$ es que, para cualquier relación causal $r$ en que un conjunto de condiciones $c$ produce un efecto $e$, (a) $r$ es necesaria y su necesidad obedece al hecho de que es imposible una situación en que se da $c$ pero no se da $e$, lo cual implica que (b) $e$ se dará en toda situación posible en que se da $c$. Estas dos tesis son conspicuas en el testimonio de Alejandro sobre $C$, en $F, 13 .{ }^{18}$ Cuando $C$ pretende demostrar que 'ninguna de las cosas producidas por cada uno de los seres de acuerdo con su naturaleza propia, puede ser de otro modo', la teoría ilustra (a) y (b) mediante un ejemplo. En primer lugar, 'ni siquiera la piedra, en efecto, si es tirada desde una cierta altura, es capaz de no moverse hacia abajo cuando nada se lo impide'. Esto corresponde a (a) porque el texto recurre a la imposibilidad de que se dé un caso en que se satisfacen las condiciones para que la piedra caiga, pero la piedra no cae. En segundo lugar, dado el peso de la piedra, 'cuando también están presentes los factores causales externos que cooperan con la piedra a su movimiento natural, es necesario que la piedra se mueva cual debe por naturaleza'. Esto, por su parte, corresponde a (b). Conviene aquí citar la parte del texto griego donde figuran estos pasajes $(F, 181,18-30)$ con el fin de dejar al lector apreciar los complejos matices conceptuales que podrían perderse en la traducción:

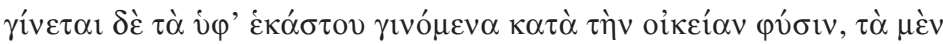

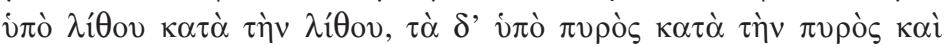

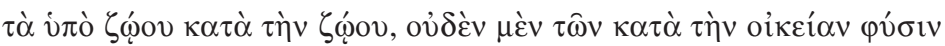

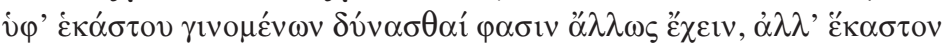

${ }^{18}$ Más adelante, cito el texto de Alejandro. Para el caso de Nemesio, cf. $N$, 105, 18-21: "Cuando se dan las mismas causas circundantes, como afirman ellos, es totalmente necesario que las mismas cosas se den, y, debido al hecho de que estas cosas fueron asignadas de este modo desde la eternidad, no es posible que en un momento determinado se den de este modo y, en otro, de otro" ( $\tau \hat{\omega} v$

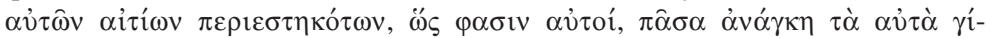

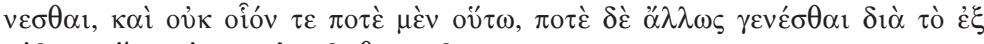

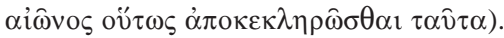




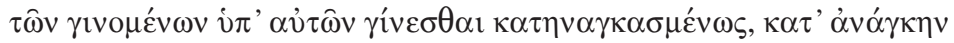

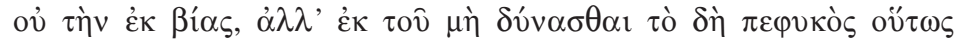

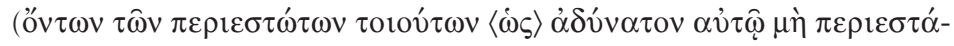

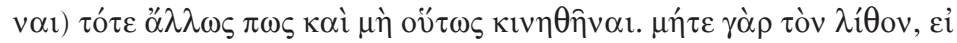

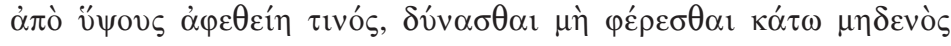

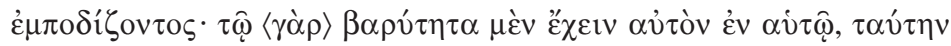

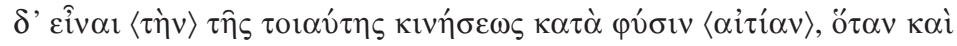

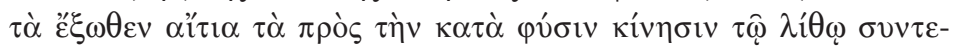

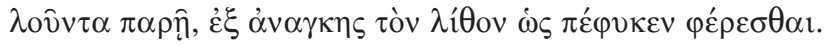

las cosas producidas por cada uno de los seres, se producen según su naturaleza propia: las producidas por la piedra, según la naturaleza de la piedra; las producidas por el fuego, según la naturaleza del fuego, y las producidas por el ser vivo, según la naturaleza del ser vivo. Afirman que ninguna de las cosas producidas por cada uno de los seres de acuerdo con su naturaleza propia, puede ser de otro modo, sino que cada una de las cosas que ellos producen ocurre necesariamente y de acuerdo con la necesidad que procede, no de la coacción, sino del hecho de que no es posible que lo que está naturalmente constituido de determinada manera, pueda, en cierto sentido, ser movido de alguna otra forma y no de ésta al darse unas circunstancias tales que resulta imposible que no se den en ese momento. Ni siquiera la piedra, afirman, si es tirada desde una cierta altura, es capaz de no irse hacia abajo cuando nada se lo impide. En efecto, en virtud de que tiene peso en sí misma (y ésta es la causa de este tipo de movimiento natural), cuando también están presentes los factores causales externos que cooperan con la piedra a su movimiento natural, necesariamente la piedra se mueve, como le corresponde por naturaleza.

Ahora bien, también es posible encontrar en la doctrina del eterno retorno una versión de (a) y (b). En efecto, según esta doctrina, si un conjunto de condiciones $c$ produjo un efecto $e$ en el ciclo cósmico presente, entonces (a') la producción causal de $e$ por $c$ es necesaria en virtud de la imposibilidad de un ciclo cósmico en que se da $c$ pero no $e$; esto, a su vez, implica que (b') e tendrá que darse en todo ciclo cósmico futuro posible en que se da $c$. Conviene recordar que, en este 
caso, el principio de regularidad 'a las mismas causas, los mismos efectos' no es hipotético. La idea no es que, si las mismas causas llegaran a repetirse, traerían consigo los mismos efectos, sin importar que dichas causas efectivamente vuelvan a repetirse. La idea es, más bien, que cuando las mismas causas vuelvan a repetirse en el próximo ciclo siguiente (en el ententido de que sí volverán a repetirse), producirán los mismos efectos. ¿Es el carácter no hipotético del principio de regularidad en la doctrina del eterno retorno una diferencia en esa doctrina y $C$ ? La pregunta amerita una respuesta cuidadosa. En efecto, es posible que $C$ misma se esté refiriendo a regularidades transcíclicas y, por ende, no-hipotéticas. ${ }^{19}$ Por este motivo, sería erróneo limitarse a suponer que el principio de regularidad que hallamos en $C$ es hipotético y, por tanto, distinto del que podemos hallar en el estoicismo temprano de la doctrina del eterno retorno.

Pasemos ahora a la segunda diferencia sustantiva entre $C$ y $C^{*}$.

(2) C ofrece una definición del concepto de 'lo que depende de nosotros' ( $\tau$ ò $\dot{\varphi} \varphi$ ' rales, qué condiciones debe satisfacer una actividad para depender de alguien: 'Afirman que depende de nosotros lo que

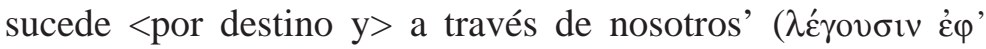

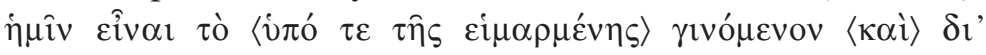
$\dot{\eta} \mu \hat{\omega} v){ }^{20}$ Esta definición usa los términos $\dot{\varepsilon} \varphi$ ' $\dot{\eta} \mu \hat{v} v$ y $\delta \mathrm{r}^{\prime} \dot{\eta} \mu \hat{\omega} v$ en un sentido técnico que no tiene ningún equivalente en $C^{*}$. En $C^{*}$ encontramos, más bien, una aplicación de la expresión 'depende de nosotros' (in nostra potestate) a casos particulares de actividad, sin estar nunca acompañada de una definición de carácter general en la que se emplean conceptos técnicos. Pero,

\footnotetext{
${ }^{19}$ Para una lectura transcíclica, cf. Long (1971), 188-189. Véase también Bobzien (1998.2), 373-374.

${ }^{20}$ Cf. F, 181, 14.
} 
una vez más, esta diferencia no representa ningún desacuerdo entre las dos teorías. Veamos por qué.

Para empezar, el uso de la expresión 'a través de nosotros' (empleada en la definición que ofrece $C$ de lo que depende de nosotros) con la connotación de responsabilidad moral no es una novedad que sólo haya surgido en la antigüedad media. Ya en Aristóteles se usa con ese sentido. ${ }^{21}$ Por otra parte, si bien $C$ usa esa expresión en un sentido técnico, lo cual no ocurre en el caso de Aristóteles (éste no define la noción de algo que sucede a través de nosotros en términos de nociones básicas), ese uso técnico puede deberse, no a la teoría original, sino a Filópator, quien decide describir la teoría de una forma sistemática, pero posiblemente ajena a su formulación original. ${ }^{22}$ De esto, sin embargo, no se sigue que $C$ sea ajena a Crisipo. En efecto, la formulación técnica de $C$ que encontramos en Alejandro y Nemesio podría ser simplemente una formalización por parte de Filópator de ideas que pertenecían originalmente a Crisipo, del mismo modo en que observamos en la historia de la ciencia moderna que ciertos científicos formalizan teorías anteriores sin proponerse añadir a esas teorías ideas sustantivas nuevas.

(3) La tercera gran diferencia entre $C$ y $C^{*}$ radica en el modo en que cada una especifica cuál es la causa interna de aquellas actividades que dependen de nosotros. En $C^{*}$, la naturaleza de la causa interna se especifica mediante una analogía con el caso del cilindro. Según el testimonio de Cicerón en $D F, 43$, el poder que tiene el cilindro de rodar equivale a la

\footnotetext{
${ }^{21} \mathrm{Cf} . E N, 1111 \mathrm{~b} 23-4,26,1112 \mathrm{a} 30-\mathrm{b} 4, \mathrm{~b} 27$. En estos pasajes, lo que ocurre 'a través de' nosotros es aquello acerca de lo cual deliberamos (o debemos de deliberar) y, por lo tanto, aquello de lo cual somos responsables. Esto lo reconoce Bobzien en (1998.1), 168 y n. 63.

${ }^{22}$ Como lo han sugerido Sharples y Long. Cf. Sharples (1978), 254, n. 124 y (1983), 143, y Long (1970), 268. Para una postura distinta, cf. Bobzien (1998.2), 378, n. 47 y, aparentemente, Sharples (2001), 552.
} 
'fuerza' de la mente y su 'propia naturaleza' (suapte vi et natura). En la literatura reciente sobre el estoicismo, esto se ha interpretado de al menos dos maneras diferentes: como la

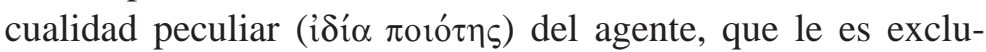
siva, o como un conjunto de propiedades que es común a un cierto tipo de personas. En cambio, en $C$, la causa interna es el uso de una capacidad que es común a todos los seres humanos por tratarse de aquello que los distingue de los demás animales. En otras palabras, para definir la causa interna, $C$ recu-

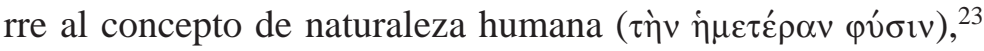
mientras que $C^{*}$ no lo hace. Esta diferencia, sin embargo, no implica que las dos teorías se contradigan entre sí. Según intentaré mostrarlo, la diferencia se debe más bien a que cada teoría pretende refutar objeciones distintas.

$C^{*}$ pretende refutar una objeción de tipo externalista. En contra de esta objeción, el objetivo de $C^{*}$ es establecer por qué el determinismo no implica que todo lo que hacemos se determina en última instancia por factores puramente externos. Para establecer esto, $C^{*}$ se propone demostrar la tesis más fuerte de que la mayor parte del comportamiento de las cosas en general no se determina de ese modo. El hecho de que $C^{*}$ establezca una analogía entre el comportamiento de los cilindros y los actos de asentimiento de los humanos, se debe justamente a la generalidad de esta tesis fuerte. Pero, si bien el eje del argumento de $C^{*}$ es esta analogía, el objetivo de esta teoría también explica por qué hace hincapié en las diferencias que existen entre objetos inanimados y las que existen entre seres humanos. En el caso de los humanos, por ejemplo, si diferentes personas reaccionan de manera diferente ante las mismas impresiones, la constitución de cada una de las primeras es relevante para explicar su reacción, de lo cual se sigue que esta reacción no puede explicarse enteramente en términos de factores externos.

\footnotetext{
${ }^{23}$ Cf. N, 106, 3-4.
} 
Pero el objetivo de $C$ es distinto. Su blanco de ataque no es una objeción de tipo externalista, sino una que afirma que la responsabilidad requiere la doble capacidad de actuar de otro modo al momento de la acción (esto es, la doble capacidad específica de actuar de otro modo), que es una noción a la que regresaré en el próximo apartado. El argumento de $C$ contra este adversario consiste en mostrar que la razón humana, bajo la forma de la capacidad de examinar impresiones, es suficiente para la responsabilidad, y que, por ese motivo, no necesitamos para ser responsables de ningún tipo de capacidad para actuar de otro modo. Dado que la capacidad de examinar impresiones no es exclusiva de ciertas personas, sino común a todos los humanos, es natural que $C$ se centre, no en diferencias al interior del género humano, como lo hace $C^{*}$, sino en la naturaleza específica de los seres humanos en general. Esto explica por qué $C$ define la causa interna en términos que definen a la naturaleza humana, sin recurrir a diferencias entre personas.

En resumidas cuentas, $C^{*}$ y $C$ sí difieren en el modo en que definen la causa interna de lo que depende de nosotros. Sin embargo, esta diferencia se debe a que pretenden refutar diferentes objeciones incompatibilistas y no existe realmente contradicción alguna entre ellas. Esto es, $C$ y $C^{*}$ son dos teorías distintas que se ocupan, de manera perfectamente complementaria, de casos distintos. Por consiguiente, no hay razón para suponer que, debido a sus diferencias, Crisipo no puede ser el autor de ambas teorías.

(4) Una cuarta diferencia, la última que examinaré aquí, es la siguiente: $C^{*}$ sostiene que la producción causal de actividades que dependen de nosotros involucra sólo un factor externo, a saber, el que 'desencadena' el proceso; $C$, en cambio, menciona dos factores externos: uno que desencadena el proceso, y otro que consiste meramente en la ausencia de obstáculos para la realización de la actividad. ¿Es esto una prueba 
de que $C$ y $C^{*}$ están en desacuerdo respecto de la producción causal de aquellas activadades que dependen de nosotros? Me propongo mostrar que no, y que esto se debe a que $C$ y $C^{*}$ se ocupan de dos tipos diferentes de actividades que dependen de nosotros.

El ejemplo que ofrece $C^{*}$-el acto de asentimientosugiere que las actividades de las cuales se ocupa esta teoría son de tipo mental. Se trata de actividades que suceden en la mente (animus) del agente. Es muy cierto que, para el asentimiento, es indispensable que el agente reciba una impresión, cuya causa suele ser externa. Pero también es cierto que un acto de asentimiento no puede ser obstaculizado por ningún suceso o estado de cosas externo. Según los estoicos, cuando estoy a punto de asentir a una impresión, no hay nada externo a mí que pudiera impedirme que lo haga. ${ }^{24}$ Mis actos de asentimiento tienen su causa suficiente en la combinación de una impresión con mi naturaleza interna. No se necesita nada más. A diferencia de $C^{*}, C$ se centra en actividades como el caminar, la cual no es una actividad mental. ${ }^{25}$ No cabe duda de que para los estoicos se necesita una serie de actividades mentales para que un agente camine. Pero caminar también requiere el movimiento de las extremidades corporales (piernas y brazos), el cual sí puede frustrarse por la presencia de obstáculos externos al agente. Por lo tanto, el hecho de que $C$ se centre en actividades cuya realización presupone el movimiento de las extremidades corporales, explica el otro hecho de que, a diferencia de $C^{*}$, ella postule la ausencia de obstáculos externos como indispensable para la realización de las actividades que dependen de nosotros.

\footnotetext{
${ }^{24}$ Esta tesis es conspicua en Epicteto. Véase en especial D, 1.6.40, 1.17.2027, 4.1.68-69. Sobre esta asunto, cf. Dobbin (1991), 132-133, y Long (2002), capítulo 8. No hay motivo para pensar que, en esto, Epicteto se aparta de los estoicos tempranos. Véase $M, 8.396-398$.

${ }^{25}$ Cf. $N, 105,10-12$.
} 
He querido mostrar en este apartado que ninguna de las diferencias principales entre $C$ y $C^{*}$ es prueba de que la primera no es crisipia. Si bien todas ellas son diferencias sustantivas, $C$ es perfectamente consistente con lo que sabemos con certeza sobre el pensamiento de Crisipo acerca de la responsabilidad moral.

\section{C y Aristóteles}

Me ocuparé ahora de una nueva razón para dudar que $C$ sea una teoría estoica tardía, como sostienen los defensores de la interpretación (C). Uno de los principales argumentos que aducen estos estudiosos es que esta teoría pretende refutar una postura anti-determinista que sólo pudo haber surgido con el aristotelismo del platonismo medio y, en concreto, hacia fines del primer siglo o principios del segundo d. C. ${ }^{26}$ Debido a esto, afirma el argumento, Crisipo no pudo haber sido el autor de $C$. Ahora bien, las razones que se mencionan a favor de la tesis de que el blanco de $C$ es un aristotélico del platonismo medio son las siguientes: (i) el blanco de $C$ sólo puede ser un anti-determinista que sostiene que la responsabilidad moral requiere la doble capacidad específica de actuar de otro modo, pero (ii) esta postura anti-determinista no pudo haber surgido antes del platonismo medio. Me parece que (i) es correcto, pero no estoy de acuerdo con (ii). En este apartado intento mostrar por qué es erróneo ese supuesto. Como veremos, la tesis de que la responsabilidad requiere la doble capacidad específica de actuar de otro modo ya está presente en Aristóteles, quien estaba perfectamente consciente del problema de la responsabilidad y el determinismo. ${ }^{27}$ Es muy posible que

\footnotetext{
${ }^{26}$ Cf. (1998.1), apartados 6 y 7 y (1998.2), 359.

${ }^{27}$ Sobre este asunto, cf. Sharples (1983), 4-7 y (1975), 264-7, así como Sorabji (1980), 245-247.
} 
los estoicos tempranos no hayan leído las obras esotéricas de Aristóteles, esto es, los tratados que actualmente pertenecen a lo que conocemos como el corpus aristotélico. Pero el hecho de que esa tesis se halle en el corpus es prueba de que el tema se debatía al interior de la escuela aristotélica en tiempos de Aristóteles. Por lo tanto, es posible que los estoicos conocieran ese debate, y que, al tomar partido, defendieran la postura que resultaba ser la opuesta de Aristóteles. Veamos, por lo tanto, por qué el concepto de doble capacidad específica ya está presente en el corpus.

La teoría de la responsabilidad de Aristóteles se desarrolla en los capítulos 2, 3 y 5 del libro tercero de la $E N$ y los capítulos 6,8 y 9 del libro dos de la $E E$. La teoría se centra en

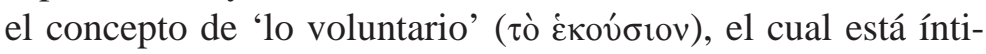
mamente ligado al de responsabilidad. De hecho, en las primeras líneas de $E N, 3.1$, los dos conceptos se presentan como equivalentes entre sí: somos responsables de una acción $\Phi$ que hemos realizado (esto es, merecemos el elogio o la censura por haberla realizado) si, y sólo si, realizamos $\Phi$ voluntariamente. El concepto aristotélico de lo voluntario contiene varias distinciones sutiles, y es, por ello, sumamente complejo. Sin embargo, uno de sus elementos centrales es sencillo. Uno actúa voluntariamente (y, por lo tanto, es merecedor de elogio o censura) sólo si dependió de uno realizar la acción o no. ${ }^{28}$ Al mismo tiempo, y como veremos ahora, Aristóteles sostiene que, para que una acción dependa de alguien, es preciso que esa persona tenga la doble capacidad de, o bien, realizar la acción, o bien, no realizarla. Veamos algunos pasajes clave.

Aunque Aristóteles no ofrece una definición formal de 'lo que depende de nosotros', él sostiene que:

En aquellos casos en los cuales depende de nosotros el actuar, también depende de nosotros el no actuar, y en los casos <en

${ }^{28}$ Cf. $E N, 1135 \mathrm{a} 23-28$ y $E E, 1225 \mathrm{~b} 7-10$ y $1226 \mathrm{~b} 30-32$. 
que depende de nosotros> el no, también <depende de nosotros> el $\mathrm{si}^{29}$

y también que:

Ciertamente, de las cosas que son posibles tanto que sean como que no sean, algunas son tales que permiten la deliberación acerca de ellas... y éstas <sc. las cosas sobre las cuales es permisible deliberar> son precisamente aquellas que depende de nosotros hacerlas o no hacerlas. ${ }^{30}$

Una interpretación posible de estas tesis es que una acción que realizo en un momento determinado depende de mí sólo si puedo no realizarla en ese momento específico. ${ }^{31}$ Pero ambos pasajes son ambiguos. En efecto, también pueden leerse en el sentido de que la doble capacidad que Aristóteles considera indispensable para la responsabilidad, no es relativa a situaciones y momentos determinados, sino que se refiere al rango total de acciones que un agente puede realizar a lo largo de su vida. La diferencia puede ilustrarse mediante un ejemplo. Si estoy en la cárcel en este momento, no tengo la doble capacidad específica de, o bien, quedarme, o bien, salir. Simplemente, tengo que quedarme donde estoy. Sin embargo, aún así puedo tener la doble capacidad general de, o bien, quedarme, o bien, salir. En efecto, supongamos que estoy cumpliendo una sentencia de sólo un año, y asumamos que no moriré durante ese año. Esto significa que me pondrán en libertad al finalizar ese año y, por lo tanto, que podré salir de la cárcel en ese momento. Por lo tanto, salir de la cárcel es algo que cae

\footnotetext{
${ }^{29}$ EN, 1113b7-8.

${ }^{30}$ EE, 1226a20-28.

${ }^{31}$ El término que el propio Aristóteles emplea para 'capacidad' en relación

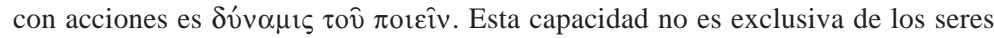
humanos, porque no presupone la racionalidad. Lo que es específicamente humano es la capacidad de actuar de otro modo. Cf. Met., $\Theta .5$ 1047b35-104.
} 
dentro del rango de acciones que puedo realizar a lo largo de mi vida (y por ello tengo la doble capacidad general de hacer algo distinto de lo que hago en el presente), aun cuando sea una acción que no puede realizar en este momento específico (y por ello carezco de la doble capacidad específica de hacer algo distinto de lo que hago en el presente). En tiempos recientes, Richard Loening fue el primer defensor de la interpretación de Aristóteles según la cual los pasajes arriba citados se refieren sólo a la capacidad general de actuar de otro modo. Siguiendo a Loening, Bobzien también la ha defendido. ${ }^{32}$ En contra de esta interpretación, pienso que hay pruebas de que para Aristóteles la responsabilidad requiere no sólo la doble capacidad general de actuar de otro modo, sino también la doble capacidad específica de hacerlo.

Algunas de las pruebas han sido ofrecidas por Richard Sorabji. ${ }^{33}$ En lo que sigue me ocuparé de otra. Se trata de un pasaje cuyas consecuencias para este asunto no han sido hasta ahora plenamente medidas. El pasaje, que citaré en un instante, figura en el capítulo 9 del De interpretatione.

Un elemento fundamental del análisis que ofrece Aristóteles en int., 9, del problema de la verdad futura, es su refutación del determinismo entendido como la tesis de que 'todo

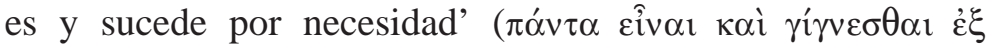

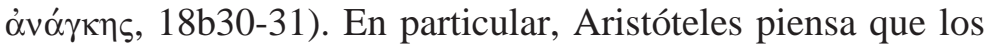
sucesos y estados futuros no están aún predeterminados y, por ello, no son inevitables. Ahora bien, es preciso advertir que el determinismo que él pretende negar acerca del futuro es de tipo específico, esto es, los sucesos y estados futuros cuya necesidad Aristóteles pone en duda no son sólo generales (e. g. habrá una batalla naval) sino también particulares. Para

\footnotetext{
${ }^{32}$ Cf. Loening (1903), capítulo 18, y Bobzien (1998.1), 140. Según Bobzien, Aristóteles es siempre ambiguo acerca de cuál es el tipo de capacidad a la que se refiere: general o específica.

${ }^{33}$ Cf. Sorabji (1980), 223-241.
} 
usar su propio ejemplo, no es ni necesario ni imposible que ocurra una batalla naval mañana. En las líneas 19a7-11 y 1819 figura una de las razones que aduce Aristóteles en contra del determinismo específico acerca del futuro:

En efecto, vemos que el principio de las cosas que serán proviene tanto de la deliberación como del realizar una acción determinada y, en general, vemos, respecto de las cosas que no están siempre en acto, el hecho de que pueden ser y <pueden> no ser; en esos casos, puede darse tanto el ser como el no ser y, por consiguiente, también puede darse tanto el ocurrir como el no

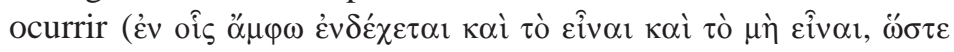

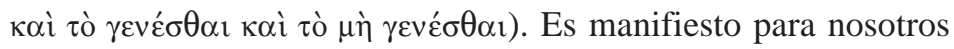
que hay muchas cosas que son de ese modo. Por lo tanto, es evidente que no todo es ni ocurre por necesidad.

Según el texto, el hecho de que exista la doble capacidad de ser y no ser, o de ocurrir y no ocurrir (como sucedería con una acción), prueba que el determinismo es falso. La necesidad en cuestión es específica, como ya vimos líneas atrás, inmediatamente antes de esta cita. Ahora bien, sabemos que si la doble capacidad a la que se refiere el texto fuera general, entonces sería compatible con el determinismo específico, como lo demuestra el ejemplo de la cárcel que ofrecí anteriormente. Como no hay motivo para suponer que Aristóteles ignora este hecho, se sigue que la doble capacidad que él pretende relacionar con la deliberación en este texto, tiene que ser específica. Esto es importante para nuestros propósitos, pues establece que, para Aristóteles, la doble capacidad que requiere la responsabilidad es la específica y no la general. En efecto, la doble capacidad que presupone la deliberación — que en int., 9 es la específica- es precisamente aquella a la que él se refiere para definir lo que depende de nosotros. ${ }^{34}$

\footnotetext{
${ }^{34}$ Según lo sugiere EN, 3.3 y su contexto. Nótese que, para Aristóteles, una acción puede depender de mí (y, por lo tanto, ser voluntaria) aunque no esté
} 
Hay una restricción en el incompatibilismo de Aristóteles. Pero no se trata de una concesión sustantiva al compatibilista. La restricción es que, al consolidarse mi carácter, puedo perder incluso la doble capacidad general de actuar de manera distinta a como actúo: hay acciones que dejan de estar en el rango de acciones que puedo realizar. ${ }^{35}$ Sin embargo, aun cuando mi carácter limite el rango de acciones que soy capaz de realizar, puedo ser responsable de las acciones que de hecho realizo. Esto sucede si tuve en mi pasado la doble capacidad específica de no realizar las acciones que de hecho formaron mi carácter. En otras palabras, mi responsabilidad por lo que hago en el presente, depende, en este caso, de que haya tenido en el pasado la doble capacidad específica para realizar acciones alternativas.

La postura de Aristóteles respecto de la responsabilidad es que ésta requiere la doble capacidad específica de actuar de otro modo. Esto lo convierte en un anti-determinista diametralmente opuesto a $C$ y, por lo tanto, en un candidato posible para ser el blanco de ataque de esta teoría estoica. De esta conclusión se desprende que es erróneo afirmar que Crisipo no pudo haber sido el autor de $C$ porque el tipo de antideterminismo que se propone rechazar sólo surgió durante la época del platonismo medio.

A lo largo de este artículo, ofrecí un examen crítico de las principales razones que aducen los defensores de una atribución tardía de la teoría estoica de la responsabilidad $C$ que figura en $F, 13$, y $N, 35$. Es cierto que no hay pruebas absolutamente concluyentes de que, en contra de lo que afirman estos autores, el autor de $C$ sea Crisipo. Sin embargo, podemos concluir que no es buena ninguna de las razones que ellos aducen a favor de una atribución tardía. En efecto, he

basada en una deliberación previa. En Aristóteles, la responsabilidad presupone la capacidad (específica) de actuar de otro modo aunque el uso de esta capacidad,

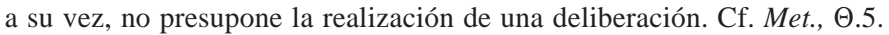

${ }^{35}$ Cf. EN, 3.5, 1114a12-21. 
mostrado que los hechos a los cuales recurren sus argumentos, o bien, son compatibles con que Crisipo sea el autor de $C$, o bien, son dudosos. En particular, la ausencia del nombre de Crisipo en $F, 13$, y la presencia del de Filópator en $N, 35$, es compatible con la autoría de Crisipo, así como lo son las diferencias, por más sustantivas que sean, entre $C$ y $C^{*}$. En cambio, es muy dudoso que $C$ haya sido diseñada para refutar a un aristotélico anti-determinista del platonismo medio, lo cual, de ser correcto, sí implicaría que Crisipo no puede ser el autor de la teoría. Ahora bien, si Crisipo fue realmente el autor de $C$ y ésta iba efectivamente dirigida contra Aristóteles, resta explicar más detalladamente esta interacción entre estos filósofos y, en especial, cómo funcionó la transmisión del aristotelismo durante la época helenística temprana. ${ }^{36}$

\section{BIBLIOGRAFÍA}

\section{Obras antiguas}

Ax, W., Ciceronis de divinatione, de fato, Timaeus, Leipzig, 1938.

Bruns, I., Alexander von Aphrodisias, De fato ad imperatores $(=F)$. Supplementum Aristotelicum 2.2., Commentaria in Aristotelem Grae$c a$, Berlin, 1892. Reimp. por R. W. Sharples con un nuevo aparato crítico en Sharples (1983) [cf. 'Obras modernas'].

Bywater, I., Aristotelis ethica Nicomachea $(=$ EN), Oxford, 1894. Varias reimps.

Marache, R., Aulu Gèlle, Nuits Attiques (= NA), Paris, 1978.

Minio-Paluello, L., Aristotelis categoriae et liber de interpretatione (= int.), Oxford, 1949. Varias reimps.

Morani, M., Nemesii Emesenii de natura hominis (= N), Leipzig, 1987. Todd, R. B., Alexander of Aphrodisias on Stoic Physics. A study of the De mixtione (= mixt.), Leiden, 1976.

${ }^{36}$ La investigación para este artículo se realizó en el marco de los proyectos PAPIIT IN 407705 y CONACYT 40891, a quienes deseo agradecer su apoyo. La penúltima versión del trabajo fue leída con extremo cuidado por un dictaminador anómino y el Director de la revista, con quienes estoy muy agradecido por sus valiosas observaciones respecto del argumento filosófico y de las traducciones. 
von Arnim, H., Stoicorum veterum fragmenta $(=S V F)$. Cuarto volumen compilado por M. Adler (y publicado en 1924), Leipzig, 1903-1905.

Walzer, R. R., \& J. M. Mingay, Aristotelis ethica Eudemia, Oxford, 1991.

\section{Obras modernas}

Amand, D., Fatalisme et Liberté dans l'Antiquité Grecque. Recherches sur la survivance de l'argumentation morale antifataliste de Carnéade chez les philosophes grecs et les théologiens chrétiens des quatre premiers siècles, Amsterdam, 1973.

BobziEn, S., "The inadvertent and late birth of the free-will problem", Phronesis, 43, 1998.1, pp. 133-175.

—, Determinism and Freedom in Stoic Philosophy, Oxford, 1998.2.

Bотros, S., "Freedom, causality, fatalism and early Stoic philosophy", Phronesis, 30, 1995, pp. 274-304.

Dobbin, R., "Prohairesis in Epictetus", Ancient Philosophy, 11, 1991, pp. 111-135.

Frede, D., "The Dramatization of Determinism: Alexander of Aphrodisias' de fato”, Phronesis, 27, 1982, pp. 276-298.

Gould, J. B., "The Stoic conception of fate", Journal of the History of Ideas, 35, 1974, pp. 17-32.

Inwood, B., Ethics and Human Action in Early Stoicism, Oxford, 1985.

Loening, R., Die Zurechnungslehre des Aristoteles, Jena, 1903.

Long, A. A., "Stoic determinism and Alexander of Aphrodisias De Fato (i-xiv)", Archiv für Geschichte der Philosophie, 52, 1970, pp. 247-268.

_, "Freedom and determinism in the Stoic theory of human action", in his Problems in Stoicism, London, 1971.

—, Epictetus. A Stoic and Socratic Guide to Life, Oxford, 2002.

ReEsor, M. E., "Necessity and fate in Stoic philosophy", in J. M. Rist (ed.), The Stoics, Berkeley-Los Angeles-London, 1978.

SAllES, R., "Determinism and recurrence in early Stoic thought", Oxford Studies in Ancient Philosophy, 24, verano 2003, pp. 253-272.

Sharples, R. W., "Aristotelian and Stoic Conceptions of Necessity in the De Fato of Alexander of Aphrodisias", Phronesis, 20, 1975, pp. 247-274.

-, "Alexander of Aphrodisias, De Fato: some parallels", Classical Quarterly, 28, 1978, pp. 243-266.

-, Alexander of Aphrodisias. On Fate, text, transl. and comm., London, 1983.

—, Cicero, On Fate (De Fato) \& Boethius, The Consolation of Philosophy iv.5-7, v (Philosophiae Consolationis), Warminster, England, 1991. 
Sharples, R. W., "Schriften und Problemkomplexe zur Ethik", en P. J. Wiesner (hrsg.), Der Aristotelismus bei den Chriechen 3: Alexander von Aphrodisias, Berlin, 2001.

Sorabi, R., Necessity, Cause, and Blame. Perspectives on Aristotle's Theory, London, 1980.

Theiler, W., "Tacitus und die antike Schicksalslehre", en Phyllobolia fur P. von der Mühl, Basel. Reimpreso en sus Forschungen zur Neuplatonismus, Berlin, 1966.

Thillet, P., Alexandre d'Aphrodise. Traité du Destin, Paris, 1984. 